\title{
Rapid Assembly of Complex 3D Siloxane Architectures
}

\author{
David B. Thompson and Michael A. Brook* \\ Department of Chemistry, McMaster University, 1280 Main Street West, \\ Hamilton, Ontario, Canada, L8S 4M1.
}

\section{Supplementary Information}

\section{Experimental}

\section{Materials and methods}

Tetramethoxysilane, tetraethoxysilane, tetrapropoxysilane, 1,1,1,3,5,5,5heptamethyltrisiloxane and tris(pentafluorophenyl)borane were purchased from Aldrich. Pentamethyldisiloxane and phenyl(tris(hydridodimethylsilyloxy))silane were purchased from Gelest. Deuterated chloroform was purchased from Cambridge Isotopes. All of the preceding chemicals were reagent grade and used without further purification. Solvents were purchased from Caledon and purified using pressurized activated alumina columns.

${ }^{1} \mathrm{H},{ }^{13} \mathrm{C}$ and ${ }^{19} \mathrm{~F}$ NMR were recorded using a Bruker AV200 spectrometer (at $200 \mathrm{MHz}$ for $\mathrm{H}, 50 \mathrm{MHz}$ for $\mathrm{C}$ and $188 \mathrm{MHz}$ for $\mathrm{F}$ ) and ${ }^{29} \mathrm{Si}$ NMR were recorded using a Bruker DRX500 spectrometer (at $99 \mathrm{MHz}$ ). ${ }^{1} \mathrm{H},{ }^{13} \mathrm{C}$ spectra are referenced to residual solvent protons. ${ }^{19} \mathrm{~F}$ and and ${ }^{29} \mathrm{Si}$ spectra are unreferenced; for ${ }^{29} \mathrm{Si}$ NMR samples, $\mathrm{Cr}(\mathrm{AcAc})_{3}$ $(\sim 0.1 \%)$ was used as a spin relaxation agent. CI-TOF-MS were collected using a Micromass GCT. MALDI-TOF mass spectra were obtained using a Waters Micromass MALDI Micro MX MADLI-TOF mass spectrometer operated in reflectron mode. Samples were dissolved in THF and dithranol was used a matrix.

\section{Synthesis of tetrakis(pentamethyldisilyloxy)silane 6: Comparison of outcomes from different tetraalkoxysilanes}

Three reactions were performed in order to determine the effect of steric environment on yield of 6. Approximately equimolar amounts of tetramethoxysilane $3(0.2772 \mathrm{~g}, 1.821$ $\mathrm{mmol})$, tetraethoxysilane $4(0.3679 \mathrm{~g}, 1.766 \mathrm{mmol})$ and tetrapropoxysilane $5(0.4681 \mathrm{~g}$, $1.770 \mathrm{mmol})$, respectively, were added to 3 separate round bottomed flasks equipped with magnetic stir bars. Pentamethyldisiloxane $(3.00 \mathrm{~mL}, 26.6 \mathrm{mmol})$ was added to each flask, and the resulting mixture dissolved in hexanes $(4 \mathrm{~mL})$ with stirring.

Tris(pentafluorophenyl)-borane $(0.40 \mathrm{mg}, 0.00078 \mathrm{mmol}$ in $10 \mu \mathrm{L}$ toluene solution) was added, and the reactions were allowed to stir at room temperature for $30 \mathrm{~min}$. Excess solvent and starting material were removed in vacuo, providing pure 6 by ${ }^{29} \mathrm{Si} \mathrm{NMR}$.

From 3 - 0.9646g, $1.415 \mathrm{mmol} ; 77 \%$ yield.

From $4-1.1592 \mathrm{~g}, 1.701 \mathrm{mmol} ; 96 \%$ yield

From 5 - 1.1735g, 1.722 mmol; 97\% yield

For characterization, see below. 


\section{Synthesis of tetrakis(pentamethyldisilyloxy)silane 6 (with catalyst removal)}

Tetrapropoxysilane $(0.5832 \mathrm{~g}, 2.205 \mathrm{mmol})$ was added to a round bottomed flask equipped with a magnetic stir-bar. Pentamethyldisiloxane $(4.00 \mathrm{~mL} ; 35.5 \mathrm{mmol})$ was added, and the resulting mixture was diluted in hexanes $(5 \mathrm{~mL})$.

Tris(pentafluorophenyl)borane solution $(0.28 \mathrm{mg}$; $0.00055 \mathrm{mmol}$ in $7 \mu \mathrm{L}$ toluene) was added with stirring. After an induction period of approximately five minutes, heat was produced, and gas vigorously evolved from solution. The mixture was stirred at room temperature for 20 minutes prior to the addition of neutral alumina (approx 1g). The resulting slurry was stirred for a further 20 minutes, after which the alumina was removed via filtration. Excess solvent and starting material were removed in vacuo, affording pure tetrakis(pentamethyldisilyloxy)silane (1.4561 g, $2.137 \mathrm{mmol}, 97 \%$ yield).

${ }^{1} \mathrm{H} \mathrm{NMR}\left(\mathrm{CDCl}_{3}\right) \delta=0.106(\mathrm{~s}, 36 \mathrm{H}), 0.088(\mathrm{~s}, 24 \mathrm{H})$

${ }^{13} \mathrm{C} \mathrm{NMR}\left(\mathrm{CDCl}_{3}\right) \delta=2.02,1.18$

${ }^{19} \mathrm{~F} \mathrm{NMR}\left(\mathrm{CDCl}_{3}\right)-$ no signals visible (32 scans)

${ }^{29} \mathrm{Si} \mathrm{NMR}\left(\mathrm{CDCl}_{3}\right.$ w $\left.\mathrm{Cr}(\mathrm{AcAc})_{3}\right) \delta=7.35,-21.22,-109.68$

HRMS (CI) calculated for $\mathrm{C}_{20} \mathrm{H}_{61} \mathrm{O}_{8} \mathrm{Si}_{9}{ }^{+}\left[\mathrm{M}+\mathrm{H}^{+}\right]=681.2290$, found 681.2286

A sample of 6 (1.0010 g) was chromatographed on silica (50 g; hexanes eluent). The eluent was concentrated on a rotary evaporator and residual solvent was removed in vacuo. The resulting compound was spectroscopically identical to $\mathbf{6}$ and was recovered in high yield (0.9635 g; $96 \%$ recovery).

\section{Synthesis of tetrakis(1,1,1,3,5,5,5-heptamethyltrisilyloxy)silane 8}

Tetramethoxysilane $(1.0071 \mathrm{~g} ; 6.616 \mathrm{mmol})$ was added to a round bottomed flask equipped with a magnetic stir bar and a water jacket condenser. 1,1,1,3,5,5,5Heptamethyltrisiloxane $(10.5 \mathrm{~mL} ; 57 \mathrm{mmol})$ was added, and the resulting mixture was diluted with hexanes (15 mL). Tris(pentafluorophenyl)borane (12 mg; $0.023 \mathrm{mmol}$ in 100 $\mu \mathrm{L}$ toluene solution) was added with stirring. After a short induction period (approximately 5 minutes), heat was produced and gas was vigorously evolved from solution. The reaction mixture was stirred at room temperature for 30 minutes, followed by the addition of neutral alumina $(\sim 1 \mathrm{~g})$. The resulting slurry was stirred for a further 20 minutes, after which alumina was removed via vacuum filtration over a fritted glass disk. Residual solvent and starting materials were removed in vacuo, affording pure (1,1,1,3,5,5,5-heptamethyltrisilyloxy)silane (6.1699 g; $6.308 \mathrm{mmol} ; 95 \%$ yield).

\footnotetext{
${ }^{1} \mathrm{H}$ NMR $\left(\mathrm{CDCl}_{3}\right) \delta=0.108(\mathrm{~s}, 72 \mathrm{H}), 0.077(\mathrm{~s}, 12 \mathrm{H})$

${ }^{13} \mathrm{C} \mathrm{NMR}\left(\mathrm{CDCl}_{3}\right) \delta=1.98,-1.88$

${ }^{19} \mathrm{~F} \mathrm{NMR}\left(\mathrm{CDCl}_{3}\right)-$ no signals visible (32 scans)

${ }^{29} \mathrm{Si} \mathrm{NMR}\left(\mathrm{CDCl}_{3}\right.$ w $\left.\mathrm{Cr}(\mathrm{AcAc})_{3}\right) \delta=7.43,-66.24,-112.59$

HRMS (CI) calculated for $\mathrm{C}_{28} \mathrm{H}_{85} \mathrm{O}_{12} \mathrm{Si}_{13}{ }^{+}\left[\mathrm{M}+\mathrm{H}^{+}\right]=977.3042$, found 977.3007
} 


\section{Synthesis of ethoxytris $(1,1,1,3,5,5,5-$ heptamethyltrisilyloxy)silane 9}

Tetraethoxysilane $(2.1800 \mathrm{~g} ; 10.46 \mathrm{mmol})$ was added to a round bottomed flask equipped with magnetic stir bar and a water jacket condenser. 1,1,1,3,5,5,5-

Heptamethyltrisiloxane $(12.00 \mathrm{~mL} ; 65.85 \mathrm{mmol})$ was added, and the resulting mixture was diluted with hexanes $(20 \mathrm{~mL})$. The reaction mixture was pre-heated in an oil bath at $60{ }^{\circ} \mathrm{C}$ for 15 minutes, at which point tris(pentafluorophenyl)borane $(20 \mathrm{mg} ; 0.039 \mathrm{mmol}$, in $500 \mu \mathrm{L}$ toluene solution) was added: gas immediately evolved. The reaction mixture was stirred at $60{ }^{\circ} \mathrm{C}$ for 30 minutes, at which point it was removed from heat and neutral alumina ( $2 \mathrm{~g}$ ) was added. The resulting slurry was stirred for a further 30 minutes while cooling to room temperature, before alumina was removed via vacuum filtration over a fritted glass disk. Residual solvent and starting materials were removed in vacuo, affording pure ethoxy $(1,1,1,3,5,5,5$-heptamethyltrisilyloxy)silane $(8.0460 \mathrm{~g} ; 10.24 \mathrm{mmol}$; $98 \%$ yield).

${ }^{1} \mathrm{H} \mathrm{NMR}\left(\mathrm{CDCl}_{3}\right) \delta=3.789\left(\mathrm{q}, 2 \mathrm{H},{ }^{3} \mathrm{~J}=7 \mathrm{~Hz}\right), 1.198\left(\mathrm{t}, 3 \mathrm{H},{ }^{3} \mathrm{~J}=7 \mathrm{~Hz}\right), 0.107(\mathrm{~s}, 54 \mathrm{H})$, $0.066(\mathrm{~s}, 9 \mathrm{H})$

${ }^{13} \mathrm{C} \mathrm{NMR}\left(\mathrm{CDCl}_{3}\right) \delta=58.56,18.25,1.86,-2.12$

${ }^{19} \mathrm{~F}$ NMR $\left(\mathrm{CDCl}_{3}\right)$ - no signals visible (32 scans)

${ }^{29} \mathrm{Si} \mathrm{NMR}\left(\mathrm{CDCl}_{3} \mathrm{w} \mathrm{Cr}(\mathrm{AcAc})_{3}\right) \delta=7.68,-65.80,-104.20$

HRMS (CI) calculated for $\mathrm{C}_{23} \mathrm{H}_{69} \mathrm{O}_{10} \mathrm{Si}_{10}{ }^{+}\left[\mathrm{M}+\mathrm{H}^{+}\right]=785.2584$, found 785.2592

\section{Synthesis of branch terminal poly(dimethyl)siloxane 10}

Ethoxytris(1,1,1,3,5,5,5-heptamethyltrisilyloxy)silane 9 (0.5001 g; $0.6364 \mathrm{mmol})$ was added to a round bottomed flask equipped with a magnetic stir bar.. Hydride terminated poly(dimethyl)siloxanes (2-3 cSt; $~ 653 \mathrm{MW}$ (determined by end group analysis using proton NMR integration), $0.2034 \mathrm{~g}$; $0.3115 \mathrm{mmol}$ ) was added to the flask with stirring, and the resulting solution was diluted with dry hexanes $(5 \mathrm{~mL})$.

Tris(pentafluorophenyl)borane $(2.1 \mathrm{mg} ; 0.0041 \mathrm{mmol}$ in $50 \mu \mathrm{L}$ toluene solution) was added with stirring. Heat and gas immediately evolved from solution. The resulting mixture was stirred at room temperature for 20 minutes before addition of neutral alumina $(\sim 0.5 \mathrm{~g})$ and stirring for a further 15 minutes. The mixture was filtered and concentrated on a rotary evaporator. Residual solvent was removed in vacuo, affording branched terminal-PDMS (0.6718 g; $0.3104 \mathrm{mmol} ; 97 \%$ yield).

${ }^{1} \mathrm{H}$ NMR $\left(\mathrm{CDCl}_{3}\right) \delta=0.134(\mathrm{~s}), 0.112(\mathrm{~s}), 0.082$ (s)

${ }^{13} \mathrm{C} \mathrm{NMR}\left(\mathrm{CDCl}_{3}\right) \delta=1.95,1.23,-1.95$

${ }^{19} \mathrm{~F} \mathrm{NMR}\left(\mathrm{CDCl}_{3}\right)-$ no signals visible (32 scans)

${ }^{29} \mathrm{Si} \mathrm{NMR}\left(\mathrm{CDCl}_{3} \mathrm{w} \mathrm{Cr}(\mathrm{AcAc})_{3}\right) \delta=7.53,-21.32,-21.83,-21.98,-22.18,-66.17$, 111.87 (a trace of $\mathbf{9}$ - not separable by chromatography - remained visible in silicon NMR, but was not observed (OEt groups) in the ${ }^{1} \mathrm{H}$ NMR)

MALDI-TOF MS: Range of siloxane products found from $n=2$ to $n=15$. For most abundant component $(\mathrm{n}=4)$; calculated $\mathrm{C}_{54} \mathrm{H}_{162} \mathrm{O}_{25} \mathrm{Si}_{26} \mathrm{Na}^{+}\left[\mathrm{M}+\mathrm{Na}^{+}\right]=1964.53$, found 1964.46; calculated $\mathrm{C}_{54} \mathrm{H}_{162} \mathrm{O}_{25} \mathrm{Si}_{26} \mathrm{~K}^{+}\left[\mathrm{M}+\mathrm{Na}^{+}\right]$calculated 1980.50, found 1980.40. 


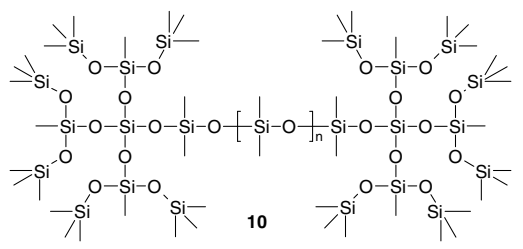

\section{Synthesis of branched siloxane 12}

Phenyl(tris(hydridodimethylsilyloxy))silane (0.2504 g; $0.7572 \mathrm{mmol}$ ) was added to a round bottomed flask equipped with a magnetic stir bar. Ethoxytris $(1,1,1,3,5,5,5-$ heptamethyltrisiloxy)silane 9 (1.7793 g; $2.2649 \mathrm{mmol})$ was added, and the resulting solution diluted in hexanes $(5 \mathrm{~mL})$. The reaction mixture was pre-heated for 25 minutes in an oil bath at $60{ }^{\circ} \mathrm{C}$, at which point tris(pentafluorophenyl)borane $(20 \mathrm{mg} ; 0.039 \mathrm{mmol}$ in $50 \mu \mathrm{L}$ toluene solution) was added. After a short induction period, gas evolved from the solution. The reaction mixture was stirred at elevated temperature for 45 minutes. Residual solvent was removed in vacuo, affording pure 12 (1.8518g; 0.7129 mmol; 94\% yield).

${ }^{1} \mathrm{H} \mathrm{NMR}\left(\mathrm{CDCl}_{3}\right) \delta=7.620(\mathrm{~m}, 2 \mathrm{H}), 7.284(\mathrm{~m}, 3 \mathrm{H}), 0.095(\mathrm{~m}, 207 \mathrm{H})$

${ }^{13} \mathrm{C} \mathrm{NMR}\left(\mathrm{CDCl}_{3}\right) \delta=134.46$ (broad), 129.47, 127.58, 1.98, 1.60, -1.89

${ }^{19} \mathrm{~F} \mathrm{NMR}\left(\mathrm{CDCl}_{3}\right) \delta=-134.71,-156.16,-163.05$

${ }^{29} \mathrm{Si} \mathrm{NMR}\left(\mathrm{CDCl}_{3} \mathrm{w} \mathrm{Cr}(\mathrm{AcAc})_{3}\right) \delta=7.49,-19.11,-66.13,-79.57,-111.62$

MALDI-TOF MS: calculated 2619.70, found 2619.69 